\title{
TRATAMENTO DA SÍNDROME DE BUDD-CHIARI POR MEIO DA COLOCAC̣ÃO DE TIPS E DE "STENT" VENOSO SUPRA-HEPÁTICO*
}

\author{
Jurandi A. Bettio ${ }^{1}$, Sílvio A. Cavazzola ${ }^{2}$, Leandro A. Scaffaro ${ }^{3}$, João Batista Petracco ${ }^{4}$, Raul \\ Ritter dos Santos ${ }^{5}$
}

Resumo OBJETIVOS: Descrever o uso do "shunt" intra-hepático portossistêmico (TIPS) e do "stent" venoso suprahepático no manejo da síndrome de Budd-Chiari, enfocando suas indicações, aspectos técnicos e benefícios do procedimento. MATERIAIS E MÉTODOS: De janeiro de 1999 a março de 2002, nove casos de síndrome de Budd-Chiari foram encaminhados ao Serviço de Hemodinâmica do Hospital São Lucas, Porto Alegre, RS. A obstrução venosa supra-hepática foi constatada em todos os casos por meio de ultra-sonografia com Doppler em cores. A criação de TIPS foi realizada entre o sistema venoso supra-hepático ou a veia cava inferior e a veia porta, posicionando-se a endoprótese entre as duas abordagens. Doppler em cores pós-procedimento foi efetuado em todos os pacientes em períodos seriados. RESULTADOS: Três casos foram tratados inicialmente com inserção de "stent" venoso por apresentarem estenose preponderante em veias supra-hepáticas. Em dois desses casos ocorreu trombose do "stent", sendo necessária colocação de TIPS. Os demais seis casos foram tratados primariamente com TIPS. Dos oito "shunts" criados, trombose da endoprótese foi constatada em três casos, resolvidas com limpeza dos trombos e dilatação com balão em um caso e inserção de novas próteses nos demais. Embolização com molas de colaterais venosas ectasiadas foi efetuada em um paciente. CONCLUSÕES: A colocação de TIPS constitui-se numa estratégia terapêutica segura e efetiva na síndrome de Budd-Chiari, promovendo uma significativa melhora clínica e hemodinâmica dos pacientes, evitando procedimentos mais invasivos e podendo, em casos sem cirrose estabelecida, servir de tratamento definitivo da hipertensão portal.

Unitermos: Síndrome de Budd-Chiari; "Shunt" portossistêmico intra-hepático (TIPS); "Stent" venoso suprahepático.

Abstract Transjugular intrahepatic portosystemic shunt (TIPS) and suprahepatic venous stenting in the management of Budd-Chiari syndrome.

OBJECTIVE: To evaluate the use of transjugular intrahepatic portosystemic shunt (TIPS) and suprahepatic venous stenting in the management of Budd-Chiari syndrome, emphasizing the indications, technical aspects and the advantages of the procedure. MATERIALS AND METHODS: Nine patients with Budd-Chiari syndrome were referred to the Hemodynamics Service of "Hospital São Lucas", Porto Alegre, RS, Brazil, for percutaneous procedure. Suprahepatic venous occlusion was detected in all cases using Doppler sonography. In the TIPS procedure, a snare loop was identified in the inferior vena cava just below the right atrium at the site of expected outflow of the occluded hepatic vein. After sonographic and fluoroscopic localization of the portal bifurcation, the needle was advanced into the portal vein and a stiff guide wire was introduced. Venography was performed and a stent was implanted. Follow-up color Doppler examinations were obtained in all cases at different intervals. RESULTS: Prominent hepatic venous stenosis was detected in three patients that were treated with suprahepatic stent implantation. Two of these patients required TIPS due to thrombosis of the stent. In the other six patients a TIPS creation was done. During follow-up, shunt dysfunction occurred in three of eight patients, requiring repeat intervention and insertion of another endoprostheses in two patients, mechanical thrombectomy and balloon dilatation in one and coil embolization of ectatic collaterals in another patient. CONCLUSION: TIPS is a safe and effective procedure for the treatment of portal hypertension caused by BuddChiari syndrome, allowing clinical and hemodynamic improvement and avoiding invasive approaches.

Key words: Budd-Chiari syndrome; Transjugular intrahepatic portosystemic shunt (TIPS); Suprahepatic occlusion.

\footnotetext{
* Trabalho realizado no Serviço de Radiologia Intervencionista do Hospital São Lucas (HSL) da Pontifícia Universidade Católica do Rio Grande do Sul (PUCRS), Porto Alegre, RS.

1. Médico Chefe do Serviço de Radiologia Intervencionista do HSL-PUCRS.

2. Médico contratado do Serviço de Radiologia Intervencionista e do Serviço de Ultra-sonografia do HSL-PUCRS.

3. Médico Residente em Radiologia Geral e Intervencionista do HSL-PUCRS

4. Médico Chefe do Serviço de Cirurgia Cardiovascular do HSL-PUCRS.

5. Médico Chefe do Serviço de Gastroenterologia do HSL-PUCRS.

Endereço para correspondência: Dr. Leandro Armani Scaffaro. Rua Pedro Pieretti, 91. Porto Alegre, RS, 90690-250.

E-mail: leandroscaffaro@yahoo.com.br

Recebido para publicação em 10/7/2002. Aceito, após revisão, em 24/9/2002.
}

\section{INTRODUÇÃO}

A síndrome de Budd-Chiari (SBC) é caracterizada pela oclusão das veias suprahepáticas, geralmente de natureza trombótica, com ou sem envolvimento associado da veia cava inferior $(\mathrm{VCI})^{(1,2)}$. O processo descrito leva a uma congestão hepática 
centrilobular progressiva, com conseqüente cirrose. $\mathrm{O}$ grau de oclusão e a presença de circulação colateral predizem a evolução clínica ${ }^{(1)}$.

As causas da SBC envolvem anormalidades da coagulação, como policitemia vera, hemoglobinúria paroxística noturna, leucemia crônica, deficiência de proteína C ou antitrombina III e uso de contraceptivos orais. Também ocorre após trauma, extensão tumoral ou anomalias congênitas obstrutivas. No entanto, nenhuma causa definida é encontrada em cerca de $60 \%$ a $70 \%$ dos pacientes ${ }^{(1-3)}$.

O diagnóstico é realizado a partir de suspeita clínica e avaliação por imagem.

A ultra-sonografia (US) mostra achados típicos, como incapacidade total ou parcial de visualização das veias supra-hepáticas, presença de material ecogênico intraluminal e indicação de estenose com dilatação proximal. Hepatomegalia está presente na maioria dos casos e ascite é invariavelmente observada. A presença e a direção do fluxo venoso hepático são melhor determinados pela US com Doppler colorido. Confirmação histológica por biópsia hepática é requerida na maioria dos $\operatorname{casos}^{(\mathbf{1 , 4})}$.

A SBC geralmente requer tratamento cirúrgico para descompressão portal ou transplante hepático ${ }^{(5)}$. Porém, nos últimos anos, o tratamento percutâneo da hipertensão portal por meio de "stents" venosos ou criação de "shunt" portossistêmico intrahepático (TIPS ) tem-se mostrado significativamente efetivo, não só para alívio temporário dos sintomas no paciente prétransplante, mas também como terapêuti- ca permanente na maioria dos $\operatorname{casos}^{(\mathbf{1 , 2 , 6})}$.

O objetivo deste estudo é descrever o uso do TIPS e do "stent" venoso supra-hepático no manejo da SBC, enfocando suas indicações, aspectos técnicos e benefícios relacionados ao procedimento.

\section{MATERIAIS E MÉTODOS}

De janeiro de 1999 a março de 2002, nove casos de SBC (sete do sexo feminino e dois do sexo masculino, idades entre 22 e 72 anos e média de 51,7 anos) foram encaminhados ao Serviço de Hemodinâmica do Hospital São Lucas, para tratamento percutâneo, Três pacientes apresentavam coagulopatias, como trombocitose (dois casos) e deficiência de antitrombina III. Nos outros seis casos não se identificaram fatores predisponentes (idiopáticos).

Todos os pacientes apresentavam hepatomegalia, dor abdominal e ascite refratária incapacitante. A obstrução venosa supra-hepática foi constatada em todos os casos através de US com Doppler em cores. Tomografia computadorizada (TC) prévia foi realizada em um caso, demonstrando trombose aguda da VCI.

A criação de TIPS foi realizada por abordagem transjugular direita com inserção de bainha 9F até a VCI. A seguir, foram adquiridas imagens com subtração digital para verificação da perviedade do sistema venoso supra-hepático e da VCI. Cateter curvo é então manipulado e inserido até a porção venosa pérvia a ser acessada. A abordagem é avançada por punção com agulha trocarte curva em direção ao hilo hepático, no sentido anterior, no intuito de cateterizar a veia porta. A seguir, a endoprótese é posicionada entre as duas abordagens, criando-se o "shunt" portossistêmico. Utilizou-se o "stent" Wallstent (Schneider) e endoprótese Miller Double Mushroom (Cook) em todos os casos.

A patência do "shunt" porto-cava e a descompressão do sistema venoso foram verificadas após o procedimento por meio de US com Doppler em cores em todos os pacientes. $\mathrm{O}$ primeiro exame foi realizado no primeiro dia após o procedimento, inclusive para verificação dos gradientes de velocidade pré e pós-"shunt". Controles subsequientes foram realizados em intervalos de uma semana, um mês, três meses e seis meses.

Até o presente momento, este estudo consta de seguimento clínico e ultra-sonográfico de três anos desde o primeiro caso.

\section{RESULTADOS}

Os nove procedimentos foram realizados de maneira efetiva, não se observando complicações relativas ao tratamento percutâneo. Encefalopatia após TIPS ocorreu em um caso, sendo controlada com medicamentos durante a internação.

Três casos foram tratados com inserção de "stent" venoso por apresentarem estenose preponderante em veias supra-hepáticas. Um deles evoluiu de maneira satisfatória, não sendo realizadas novas abordagens. Nos outros dois casos ocorreu trombose do "stent", sendo necessária colocação de TIPS (Figura 1).
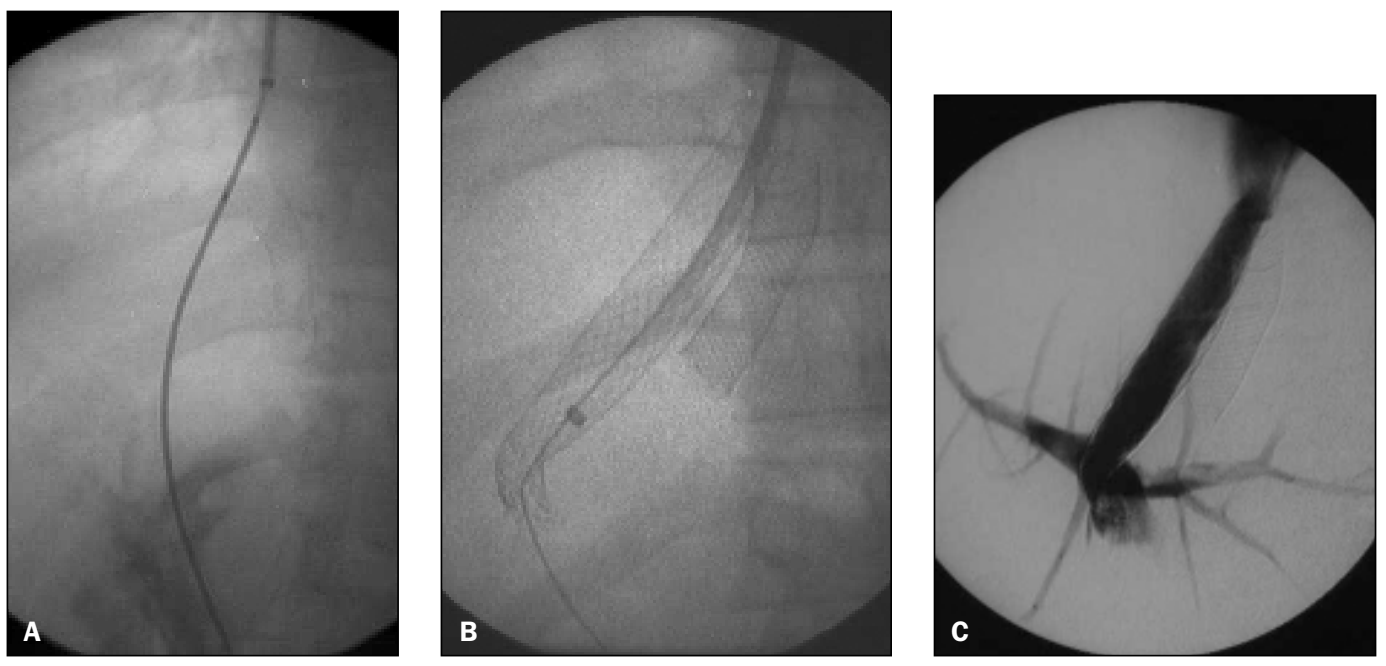

Figura 1. Caso 2. Paciente do sexo feminino, 33 anos de idade, portadora de deficiência de antitrombina III. "Stent" prévio na veia supra-hepática esquerda (A) apresentou trombose. Foi realizada inserção de TIPS (B). O controle venográfico mostra patência adequada do "shunt" porto-cava (C). 
Nos demais seis casos presenciava-se trombose importante do sistema venoso supra-hepático, sendo tratados primariamente com colocação de TIPS.

Dos oito "shunts" criados, trombose da endoprótese foi constatada em três casos, uma delas resolvida com limpeza dos trombos e dilatação da prótese com balão 12 $\mathrm{mm}$ (Figura 2). Nos outros dois casos foi necessária inserção de nova prótese, que foi efetiva para o controle definitivo da hipertensão portal em um deles.

No entanto, no outro paciente referido, a evolução clínica após o segundo TIPS não ocorreu de maneira satisfatória e um total de quatro endopróteses foram colocadas até a resolução do quadro, sendo a última inserida no interior de uma prótese previamente trombosada. Durante este último procedimento, observou-se que a presença de colaterais venosas ectasiadas junto ao "shunt" prévio favoreciam a trombogênese do sistema criado. Dessa forma, foi realizada embolização com molas dos va- sos descritos, que permitiu a evolução satisfatória do novo TIPS (Figura 3).

Em dois casos havia trombose concomitante do sistema porta, que foi tratada com limpeza com balão e injeção de estreptoquinase.

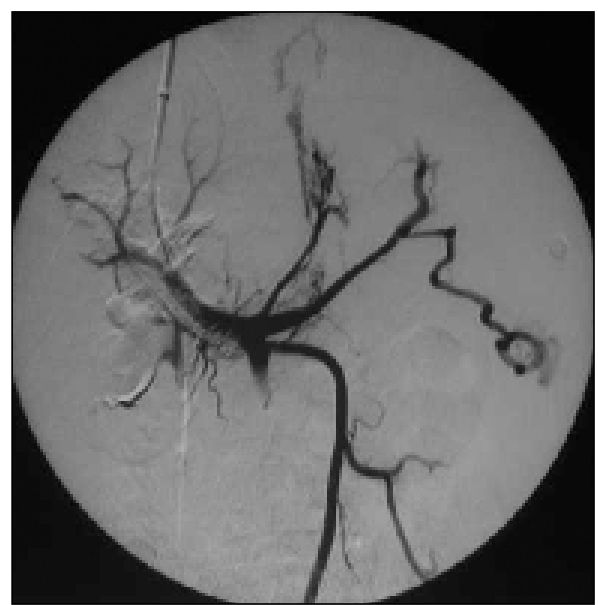

A

Figura 2. Caso 4. Controle venográfico (A) mostra obstrução parcial ao fluxo no "shunt" porto-cava. Realizada limpeza dos trombos e dilatação do TIPS com balão $12 \mathrm{~mm}$ (B).

Todos os pacientes envolvidos apresentaram melhora clínica e laboratorial, especialmente em relação à ascite, com alta hospitalar em condição assintomática.

As revisões ambulatoriais e ultra-sonográficas dos pacientes, realizadas nos inter-

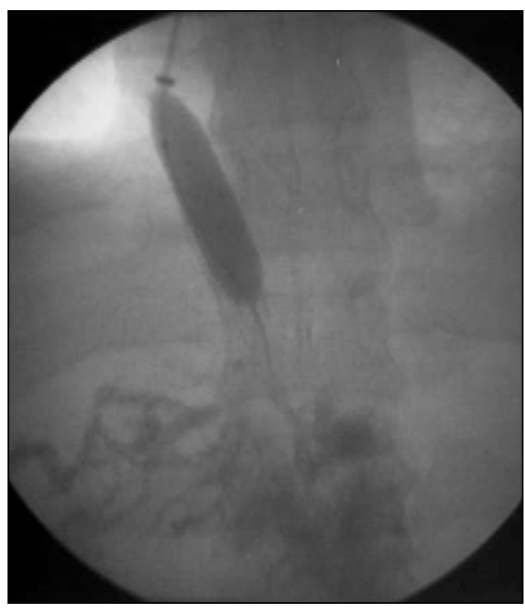

B

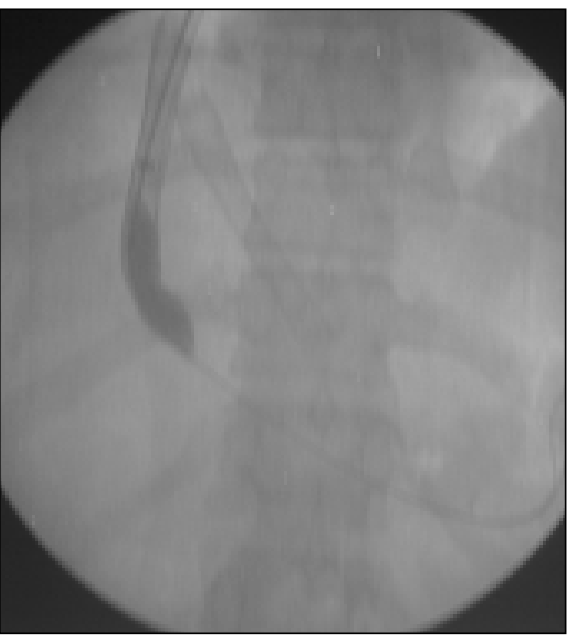

B

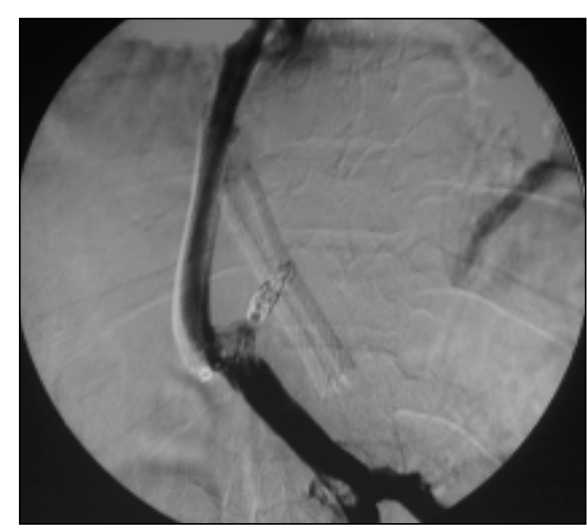

E

Figura 3. Caso 6. Paciente de 35 anos de idade, com dor abdominal. TC mostra hepatoesplenomegalia, com proeminência do lobo caudado, ascite e trombose aguda da veia cava inferior (A). Mesmo após inserção de três próteses (C), uma delas no interior da prévia (dilatada em B), não houve resolução do quadro. Controle venográfico (D) mostra presença de colaterais venosas junto ao TIPS, que favoreciam a formação de trombos e a oclusão da endoprótese. Após embolização das colaterais com molas (E), ocorreu resolução satisfatória do quadro.

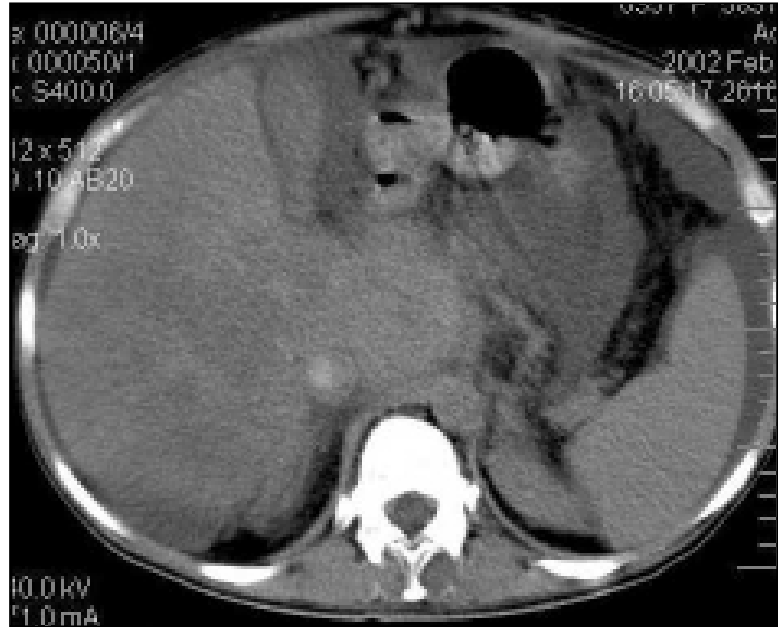

A

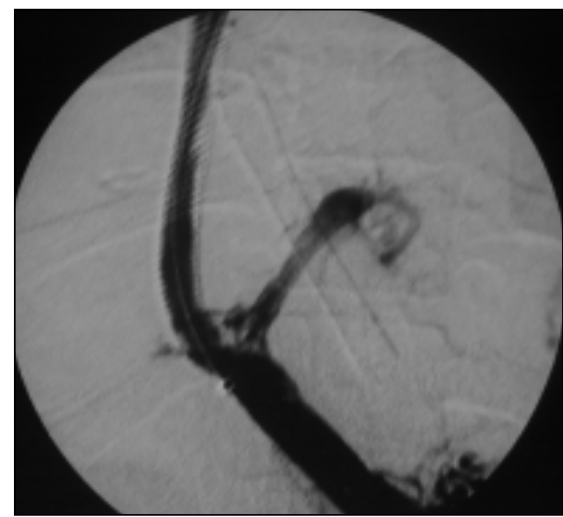

D

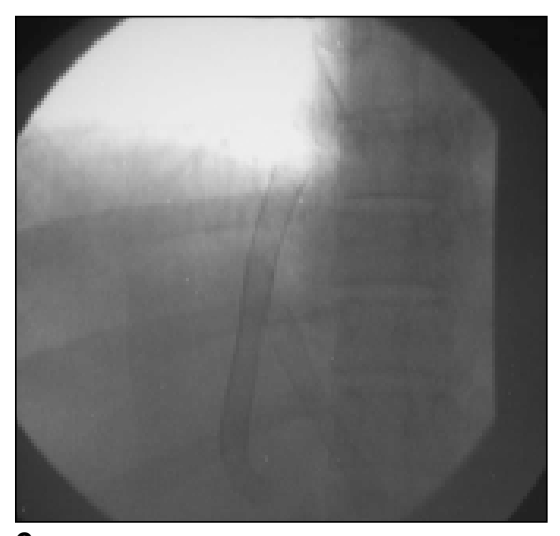

C 


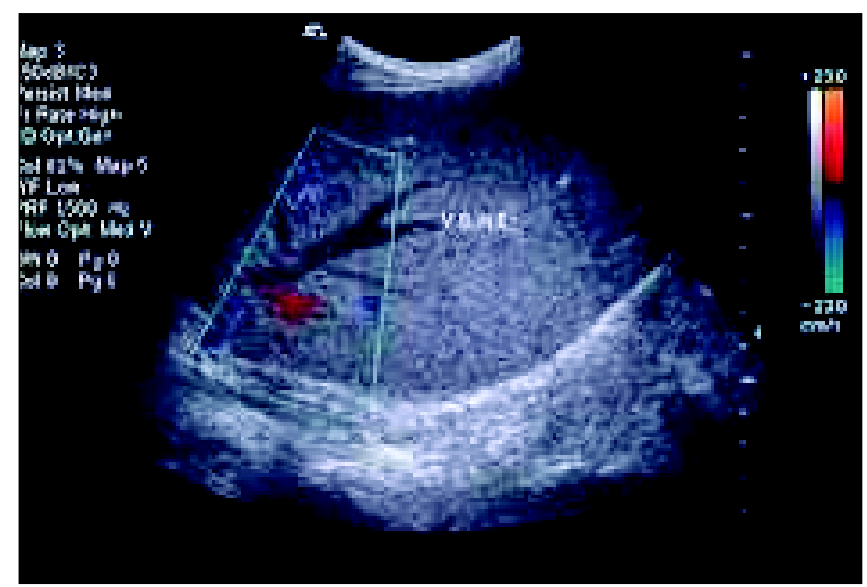

A

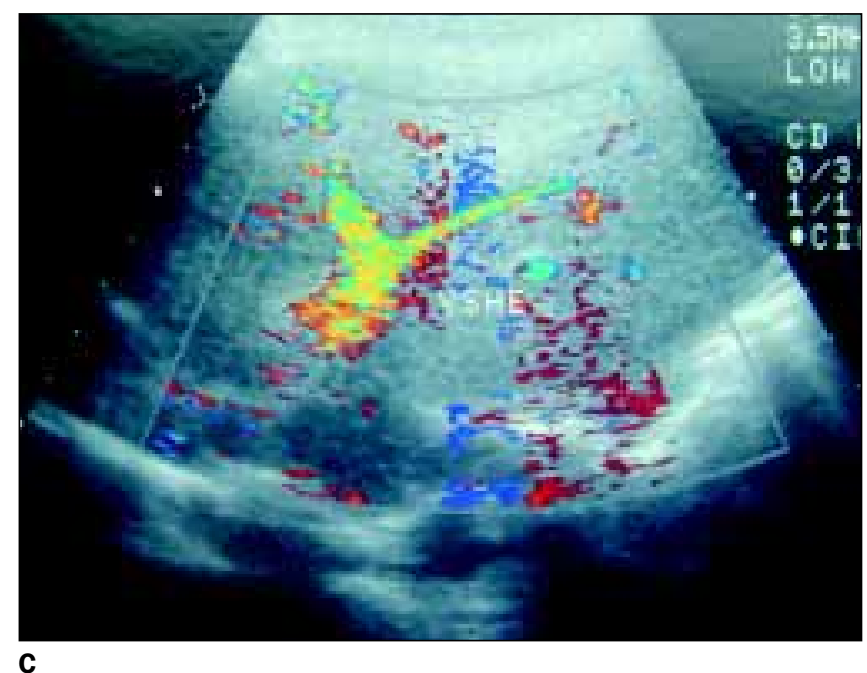

C

valos referidos, mostram adequado controle da hipertensão portal, estando todos os pacientes envolvidos com resolução permanente do quadro até o presente momento, sem a necessidade de procedimento cirúrgico adicional (Figura 4).

A Tabela 1 resume dados da casuística relatada.

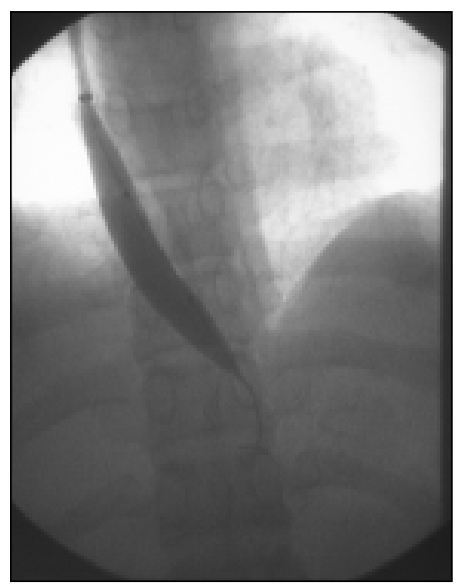

Figura 4. Caso 7. Paciente de 22 anos de idade, com síndrome de Budd-Chiari. Doppler mostrando ausência de fluxo na veia suprahepática esquerda (A). Controle ecográfico três anos após TIPS (dilatação da prótese em $\mathbf{B}$ ) mostra fluxo na supra-hepática esquerda (C), bem como na prótese e sistema porta (D).

B

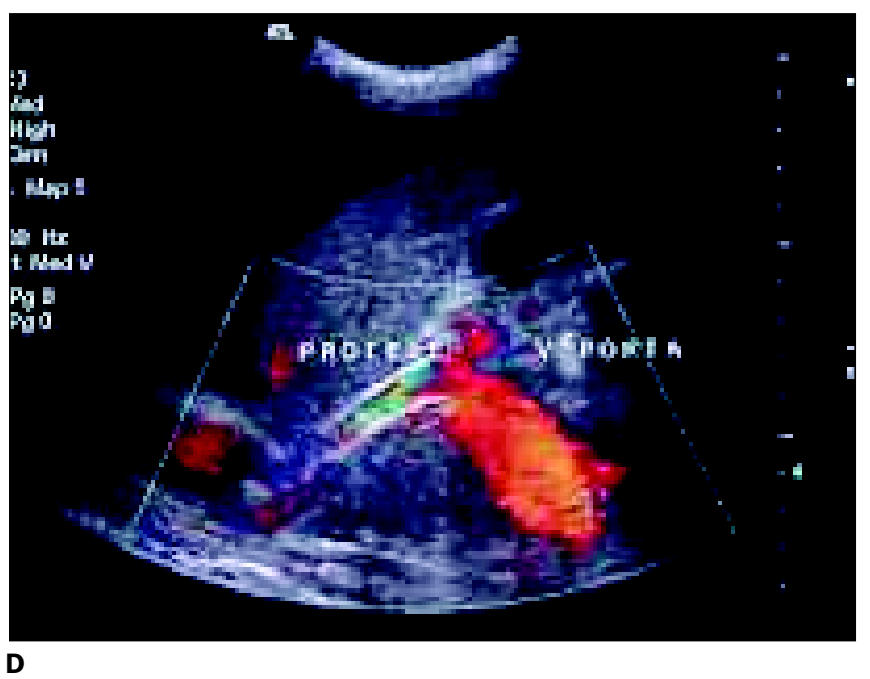

\section{DISCUSSÃO}

Inicialmente, havia muita resistência ao tratamento percutâneo da SBC, na medida em que a acelerada trombogênese neste grupo de pacientes ocluía a maioria dos "shunts" criados ${ }^{(5,7)}$. Estudos recentes corroboram o fato de que o TIPS é efetivo e seguro nesses casos, mesmo quando são necessárias reintervenções, cujo manejo é mais seguro, mais rápido e menos invasivo que as soluções cirúrgicas disponíveis ${ }^{(\mathbf{1 , 2}, \mathbf{8})}$.

Assim, observou-se que nos procedimentos em que a endoprótese é posicionada de maneira eficaz, evitando o turbilhonamento do fluxo, ou quando se elimi-

Tabela 1

\begin{tabular}{|c|c|c|c|c|c|c|c|}
\hline $\mathrm{N}$ & $\begin{array}{l}\text { Idade } \\
\text { (anos) }\end{array}$ & Sexo & Etiopatogenia & Ascite & $\begin{array}{l}\text { Hipertensão } \\
\text { porta }\end{array}$ & $\begin{array}{l}\text { “Stent" supra- } \\
\text { hepático }\end{array}$ & TIPS \\
\hline 1 & 43 & Masculino & Trombocitose & + & + & + & - \\
\hline 2 & 33 & Feminino & Deficiência de antitrombina III & + & - & + & + \\
\hline 3 & 64 & Feminino & Idiopática & + & - & - & + \\
\hline 4 & 70 & Masculino & Idiopática & + & - & - & + \\
\hline 5 & 59 & Masculino & Idiopática & + & + & - & ++ \\
\hline 6 & 35 & Feminino & Idiopática & + & + & + & +++ \\
\hline 7 & 22 & Feminino & Idiopática & + & + & - & $+^{*}$ \\
\hline 8 & 72 & Feminino & Trombocitose & + & + & - & + \\
\hline 9 & 68 & Feminino & Idiopática & + & + & - & + \\
\hline
\end{tabular}

\footnotetext{
* Após trombose do TIPS, realizou-se limpeza dos trombos e dilatação da prótese com balão 12 mm, com sucesso.
} 
nam fatores trombogênicos adjacentes, como, por exemplo, ectasia venosa colateral, o TIPS mantém-se pérvio por longos períodos $^{(2)}$. Essas situações foram constatadas em dois casos do presente estudo, nos quais a inserção de nova prótese em posição mais retilínea ou a embolização com mola de colaterais venosas foram suficientes para a regularização de fluxo, evitando-se a trombose.

Além disso, cabe ressaltar que na SBC não necessariamente há cirrose estabelecida. Dessa forma, a criação de "shunt" percutâneo pode ser tratamento definitivo nesses pacientes, evitando cirurgias ou mesmo o transplante hepático ${ }^{(2)}$.

Em suma, a colocação de TIPS ou de "stent" venoso supra-hepático constitui-se numa estratégia terapêutica segura e efeti- va na SBC, promovendo significativa melhora clínica e hemodinâmica dos pacientes, evitando procedimentos mais invasivos relacionados à anestesia geral e prolongados períodos transoperatórios. O tratamento percutâneo dessa entidade mostra aumento importante da sobrevida desses pacientes e alívio significativo de sintomas incapacitantes e pode, em casos sem cirrose estabelecida, servir de tratamento definitivo da hipertensão portal.

\section{REFERÊNCIAS}

1. Blum U, Rossle M, Haag K, et al. Budd-Chiari syndrome: technical, hemodynamic, and clinical results of treatment with transjugular intrahepatic portosystemic shunt. Radiology 1995;197:805-11.

2. Perello A, Garcia-Pagan JC, Gilabert R, et al. TIPS is a useful long-term derivative therapy for patients with Budd-Chiari syndrome uncontrolled by medical therapy. Hepatology 2002;35:132-9.
3. Ganger DR, Klapman JB, McDonald V, et al. Transjugular intrahepatic portosystemic shunt (TIPS) for Budd-Chiari syndrome or portal vein thrombosis. Am J Gastoenterol 1999;94:603-8.

4. LaBerge JM, Ring EJ, Gordon RL, et al. Creation of transjugular intrahepatic portosystemic shunt with wallstent endoprosthesis: results in 100 patients. Radiology 1993;187:413-20.

5. Ceretti AP, Intra M, Opocher E, Santambrogio R, Castrucci M, Spina GP. Application of self-expandable mettalic stents in the inferior vena cava followed by portosystemic shunt in the treatment of primary Budd-Chiari syndrome complicated by caval obstruction. Surgery 1997;122:964-6.

6. Bilbao JI, Pueyo JC, Longo JM, et al. Interventional therapeutic techniques in Budd-Chiari syndrome. Caridovasc Intervent Radiol 1997;20:112-9.

7. Slakey DP, Klein AS, Venbrux AC, Cameron JL. Budd-Chiari syndrome: current management options. Ann Surg 2001;233:522-7.

8. Michl P, Bilzer M, Waggershauser T, et al. Successful treatment of chronic Budd-Chiari syndrome with a transjugular intrahepatic portosystemic shunt. J Hepatol 2000;32:516-20. 